\title{
CYP2J2 Is a Diagnostic and Prognostic Biomarker Associated with Immune Infiltration in Kidney Renal Clear Cell Carcinoma
}

\author{
Xiong Zou $\mathbb{D}^{1,2,3,4}$ and Zengnan $M o \mathbb{D}^{1,2,3,4}$ \\ ${ }^{1}$ Department of Urology, The First Affiliated Hospital of Guangxi Medical University, 530021 Nanning, Guangxi, China \\ ${ }^{2}$ Center for Genomic and Personalized Medicine, Guangxi Medical University, 530021 Nanning, Guangxi, China \\ ${ }^{3}$ Guangxi Collaborative Innovation Center for Genomic and Personalized Medicine, 530021 Nanning, Guangxi, China \\ ${ }^{4}$ Guangxi Key Laboratory for Genomic and Personalized Medicine, Guangxi Key Laboratory of Colleges and Universities, \\ 530021 Nanning, Guangxi, China
}

Correspondence should be addressed to Zengnan Mo; mozengnan@gxmu.edu.cn

Received 14 April 2021; Revised 20 May 2021; Accepted 11 June 2021; Published 24 June 2021

Academic Editor: Nagarajan Raju

Copyright ( 2021 Xiong Zou and Zengnan Mo. This is an open access article distributed under the Creative Commons Attribution License, which permits unrestricted use, distribution, and reproduction in any medium, provided the original work is properly cited.

\begin{abstract}
Cytochrome P450 family 2 subfamily J member 2 (CYP2J2), a member of the monooxygenase cytochrome P450 (CYP) family and the only member of the human CYP2J subfamily, has many functions, including regulation of oxidative stress, inflammation, apoptosis, and immune responses. However, its role in cancer development has not been clearly elucidated. In this study, expression levels of CYP2J2 in various cancer types were determined using the Oncomine, the Gene Expression Profiling Interactive Analysis (GEIPA), DriverDBv3, UALCAN, and Tumor Immune Estimation Resource (TIMER) databases. The prognostic value of CYP2J2 for KIRC was analyzed using GEPIA, UALCAN, OSkirc, and DriverDBv3 databases. We evaluated the expression levels of CYP2J2 transcript, protein, and promoter methylation at different clinical characteristics in KIRC through the UALCAN database. Simultaneously, CYP2J2 network-related functions were evaluated using the GeneMANIA interactive tool while the biological processes involved in CYP2J2 and its interactive genes were investigated through Metascape and FunRich. Then, we used TIMER to determine the correlation between CYP2J2 expression levels and immune infiltration levels in KIRC. In KIRC, the CYP2J2 gene, RNA, and protein were found to be overexpressed. However, the methylation level of CYP2J2 promoter in KIRC was lower than in normal tissues. Surprisingly, elevated expression levels of CYP2J2 exhibited better prognostic outcomes in KIRC. Evaluation of protein-protein interaction networks and biological processes revealed that CYP2J2 was principally involved in immune responses, apoptosis, and other metabolic processes. Moreover, we found that the expression levels of CYP2J2 were positively correlated with infiltration levels of B cells, CD8 + T cells, neutrophils, and dendritic cells in KIRC. Therefore, we speculated that the overexpression of CYP2J2 prolonged the survival outcome of KIRC patients, which may be related to the change of tumor immune microenvironment. Moreover, all these new understandings of CYP2J2 may provide important value for the early diagnosis and new targeted drug therapy of KIRC.
\end{abstract}

\section{Introduction}

Renal cell carcinoma (RCC), a tumor whose origin is the renal epithelium [1], can be grouped into various subtypes based on its histological characteristics, with KIRC accounting for the vast majority of renal cell carcinoma subtypes. KIRC, a metabolic disease histologically characterized by lipid accumulation and storage [2], has been reported to be the most frequent cause of kidney cancer-associated mortal- ities [3]. Globally, KIRC is associated with 175,000 annual mortalities, with about $30-35 \%$ of patients undergoing surgery presenting with distant metastases [4]. Patients with relapsed or distant metastases of KIRC exhibit poor prognostic outcomes, with shorter median survival times of 21 months and 13 months [5], respectively. Several studies have suggested tumor lymph node metastasis (TNM) staging system is the main prognostic factor for KIRC [5-7]. However, prognostic stratification by molecular markers (such as 
Disease summary for CYP2J2

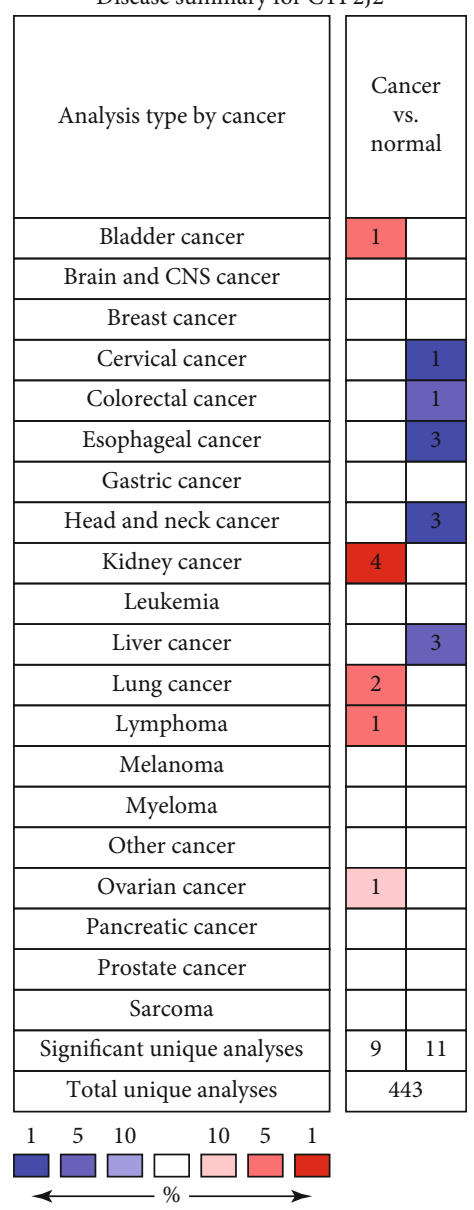

(a)

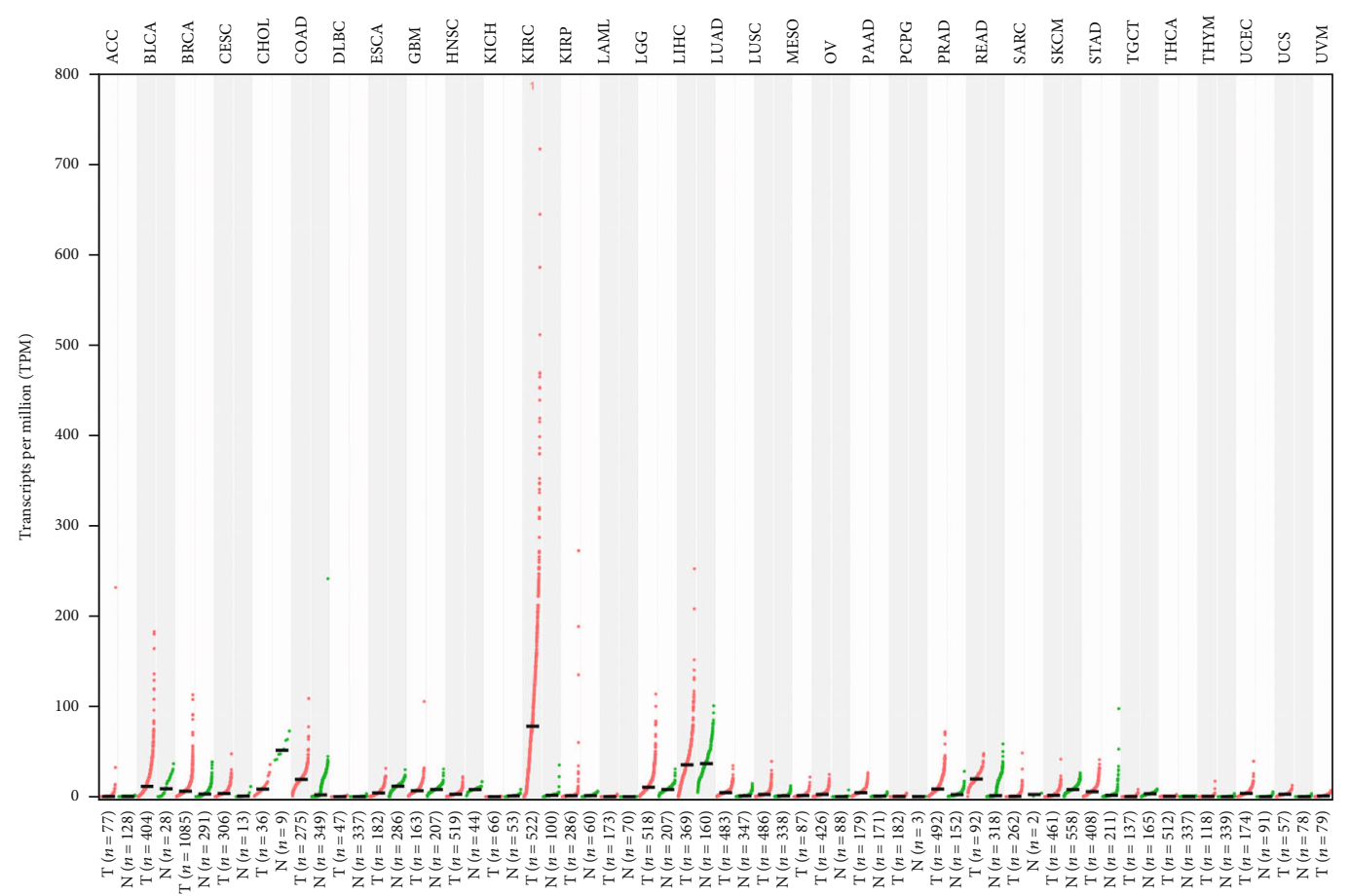

(b)

Figure 1: Continued. 


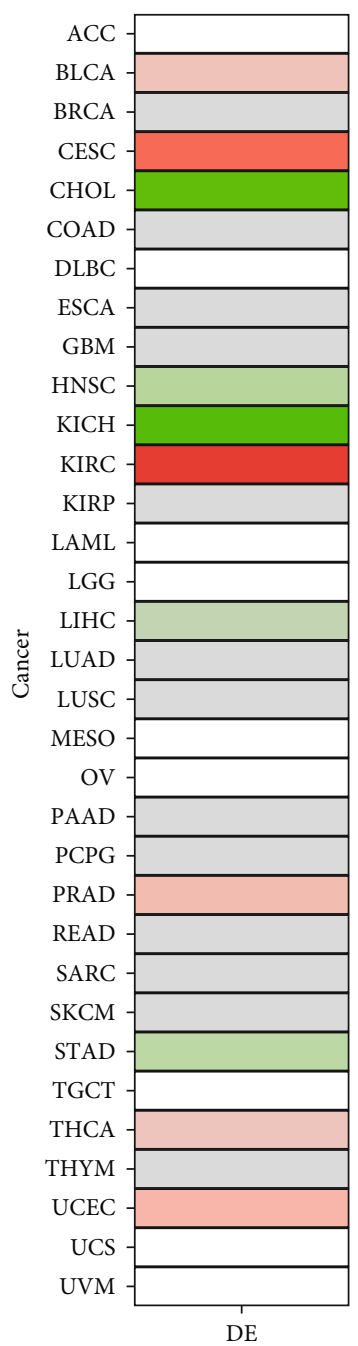

(c)

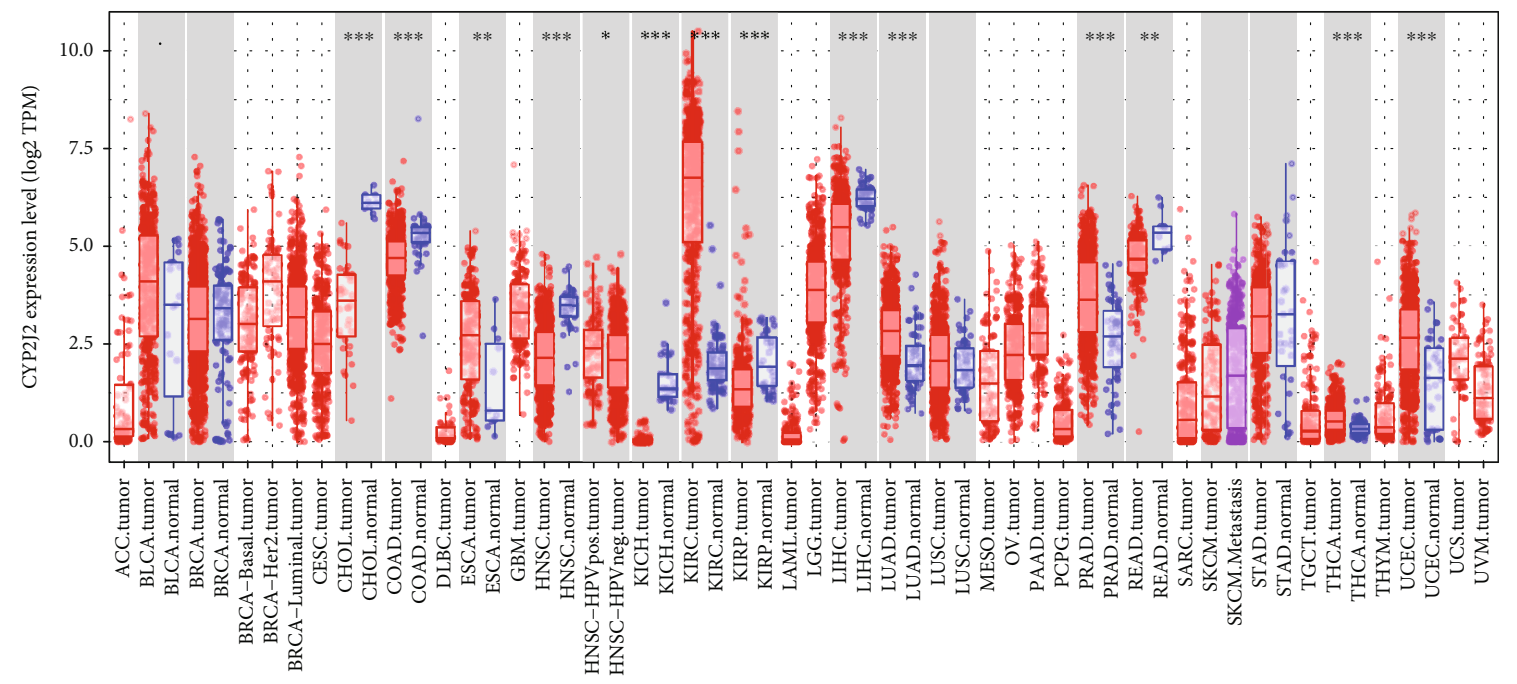

(d)

FIGURE 1: Expression levels of CYP2J2 in various human cancers. (a) Expression profiles of the CYP2J2 gene in tumor and paired normal tissue samples from the Oncomine database. (b) Expression profiles of the CYP2J2 transcript in different cancer types and paired of normal tissues from the GEPIA database. ( $c$ and d) Expression levels of the CYP2J2 gene in different cancer types compared to corresponding normal tissues from the DriverDBV3 and TIMER database. CYP2J2: Cytochrome P450 family 2 subfamily J member 2. 


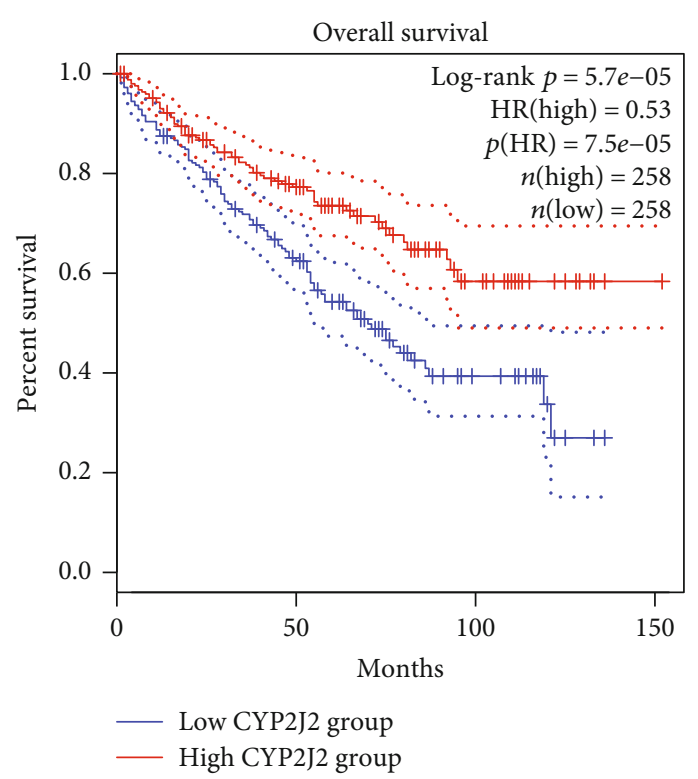

(a)

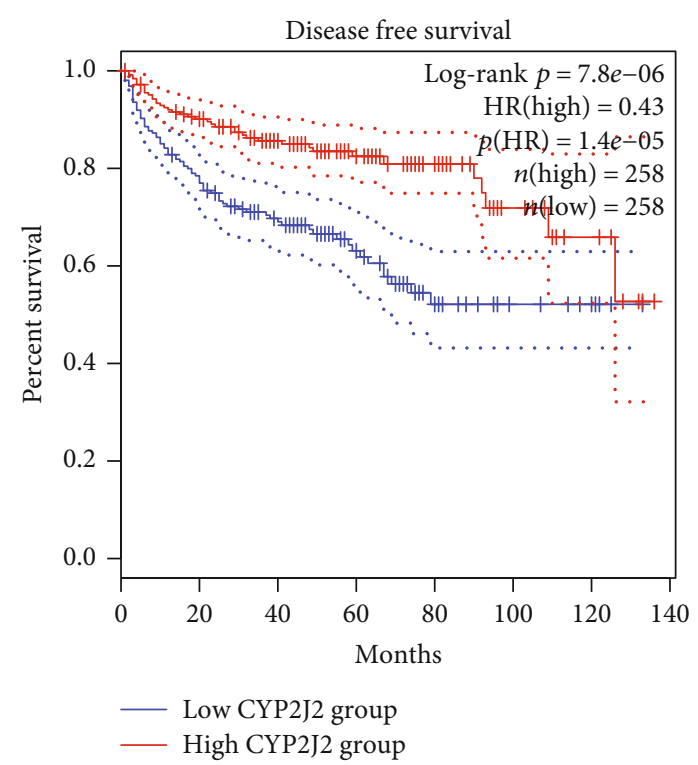

(b)

FIGURE 2: Comparisons of the effects of high and low expression levels of CYP2J2 on survival time of KIRC patients using GEPIA database. (a and b) Elevated expression levels of CYP2J2 were associated with longer OS and DFS outcomes for KIRC patients. OS: overall survival; DFS: disease free survival; KIRC: kidney renal clear cell carcinoma.

expression of specific genes) can improve the accuracy of prognostic prediction [8]. Therefore, identifying early diagnostic markers and prognostic molecular biomarkers will enhance early KIRC diagnosis, thereby informing early and active treatment.

Through the Oncomine, GEIPA, DriverDBv3, and TIMER databases, we found that CYP2J2 was differentially expressed in multiple tumors, with its expression in KIRC being most significantly elevated. The CYP2J2 gene encodes an enzyme responsible for the oxidative metabolism of various exogenous and endogenous compounds and is involved in various physiological and pathological processes in the human body [9]. It has been reported that CYP2J2 plays various roles in the human body, including anti-inflammatory [10], regulation of cardiovascular functions [11], improving metabolism [11], and in immune regulation [12]. In addition, CYP2J2 has been associated with the occurrence and development of many tumor types [13]; however, its role in KIRC pathogenesis has not been reported.

This study is aimed at evaluating the expression levels of CYP2J2 in KIRC as well as determining its value in predicting survival outcomes for KIRC patients through multiple databases, including Oncomine, GEPIA, UALCAN, OSkirc, DriverDBv3, and TIMER. In order to further speculate the possible mechanism of CYP2J2 in KIRC, we used GeneMANIA, Metascape, and FunRich to investigate the functional networks involving CYP2J2 as well as the biological processes involving CYP2J2 interactive genes. At the same time, we evaluated the relationships between CYP2J2 expression and immune cell infiltration levels in KIRC through the TIMER database. This study elucidates on the correlation between CYP2J2 expression levels and KIRC prognosis and provides a potential early diagnostic biomarker and therapeutic target for KIRC.

\section{Materials and Methods}

2.1. Oncomine. Oncomine (https://www.oncomine.org/ resource/main.html), a database dedicated to collecting and analyzing cancer-related data [14], has 715 datasets from 86733 samples [15]. This database was used to determine the expression levels of CYP2J2 in a variety of cancers using the following criteria: $p$ value of $1 E-4$, fold change as 2 , gene rank as top 10\%, and data type as all (DNA and mRNA).

2.2. GEPIA. GEPIA (http://gepia.cancer-pku.cn/) generates gene expression profiles of multiple cancer types and pairs of normal samples using the TCGA and GTEx databases [16]. GEPIA was used to determine the expression levels of the CYP2J2 gene in a variety of cancers. Moreover, we evaluated the correlation between CYP2J2 expression levels and overall survival (OS) as well as disease-free survival (DFS) for KIRC patients. Regarding the survival curve, $p<0.05$ was considered statistically significant.

2.3. UALCAN. UALCAN (http://ualcan.path.uab.edu/ analysis.html) is a website with multiple functions for analyzing and mining TCGA databases, thereby allowing users to verify the expression levels of genes in various cancer types, draw graphs describing gene expression and patient survival information, and to assess epigenetic regulation of gene expression by promoter methylation [17]. We determined the expression levels of the CYP2J2 gene, transcript, protein, and promoter methylation in KIRC and the effect of CYP2J2 on the patients' survival time through UALCAN. Regarding the survival curve, $p<0.05$ was considered statistically significant.

2.4. OSkirc. OSkirc (http://bioinfo.henu.edu.cn/KIRC Combined) is a free and fast online tool that enables users 


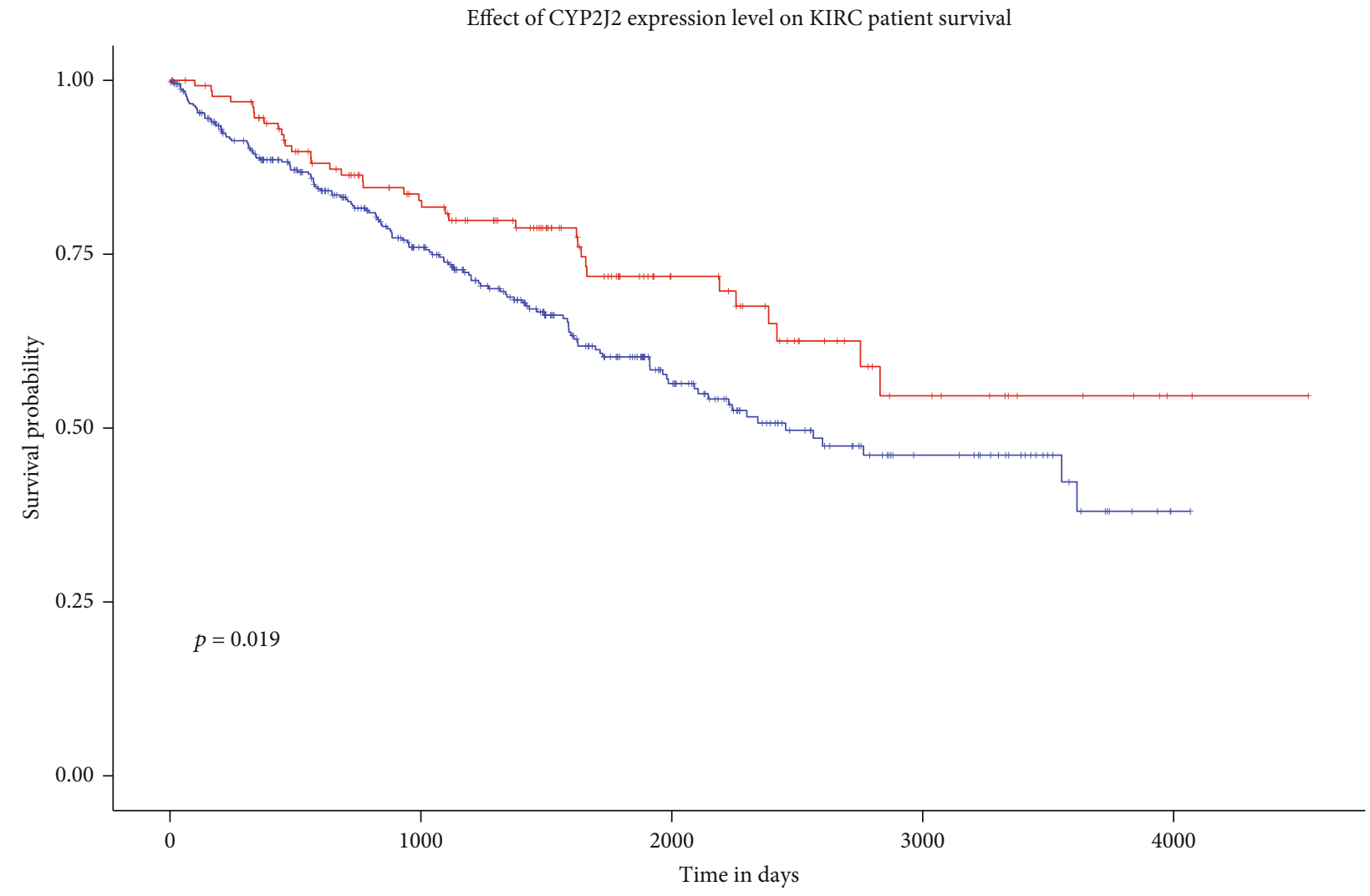

Expression level

- High expression $(n=134)$

$\longrightarrow$ Low/Medium-expression $(n=397)$

(a)

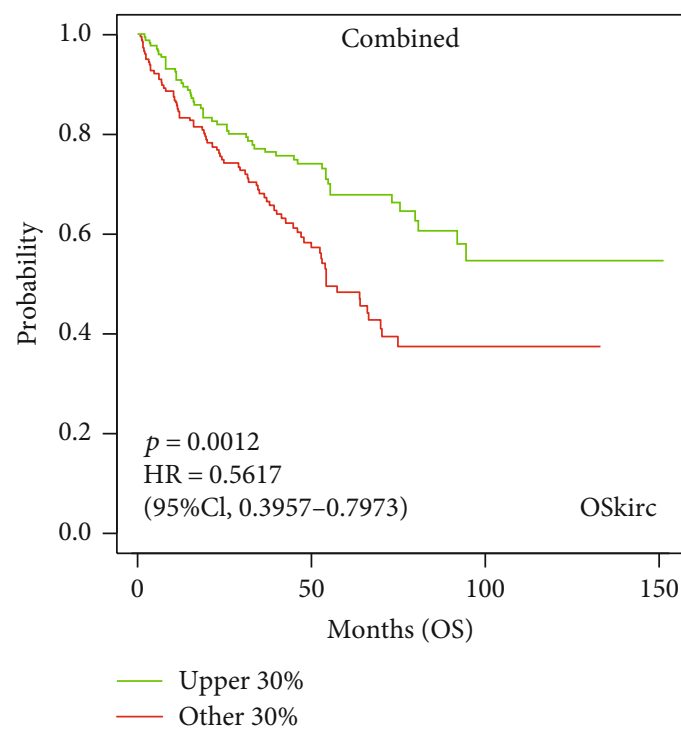

(b)

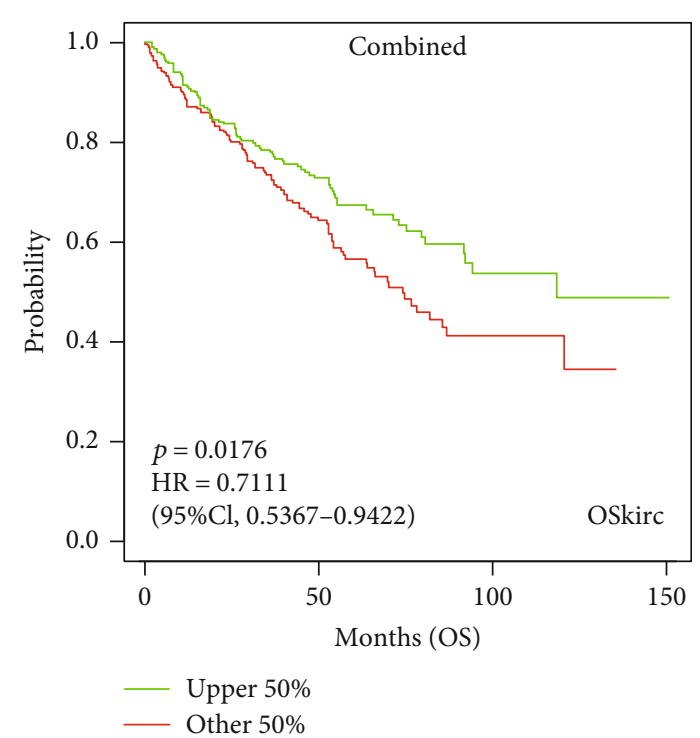

(c)

FIGURE 3: Effect of CYP2J2 expression levels in UALCAN (a) and OSkirc (b and c) database on the survival of KIRC patients. Overexpression of CYP2J2 prolonged the survival time of KIRC patients.

to easily investigate the prognostic value of genes associated with KIRC [18]. Through this database, we used the following criteria to detect the effect of CYP2J2 on the overall survival of KIRC patients: the data source from a combination of GSE22541, GSE29609, GSE3, and TCGA; split patients by upper $50 \%$ VS lower $50 \%$ and upper $30 \%$ VS lower $30 \%$. Regarding the survival curve, $p<0.05$ was considered statistically significant. 


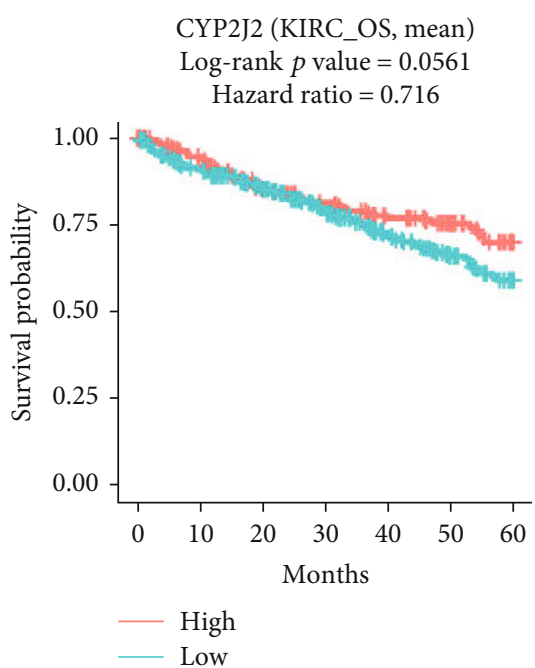

(a)

CYP2J2 (KIRC_PFI, mean) Log-rank $p$ value $=0.00448$ Hazard ratio $=0.594$

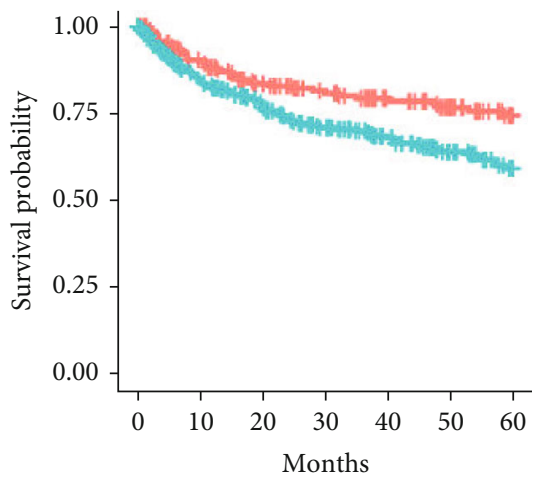

— High

(c)

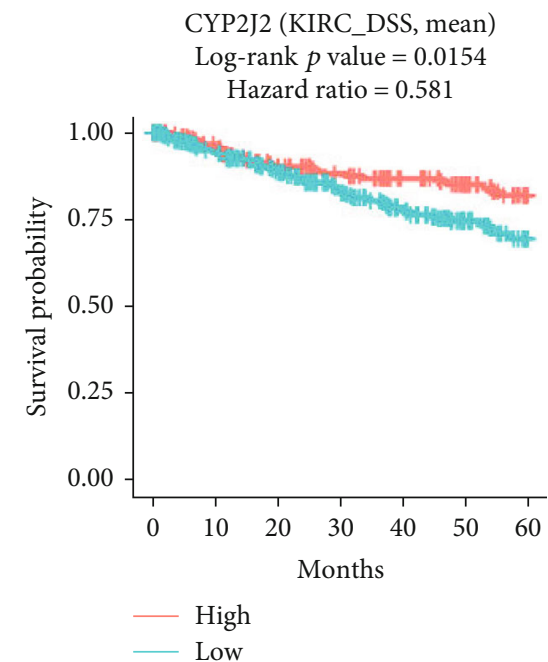

(e)

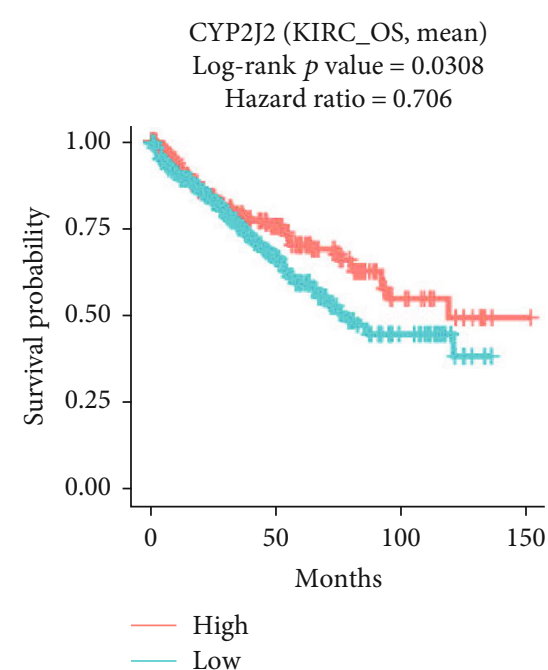

(b)

CYP2J2 (KIRC_PFI, mean)

Log-rank $p$ value $=0.00269$ Hazard ratio $=0.594$

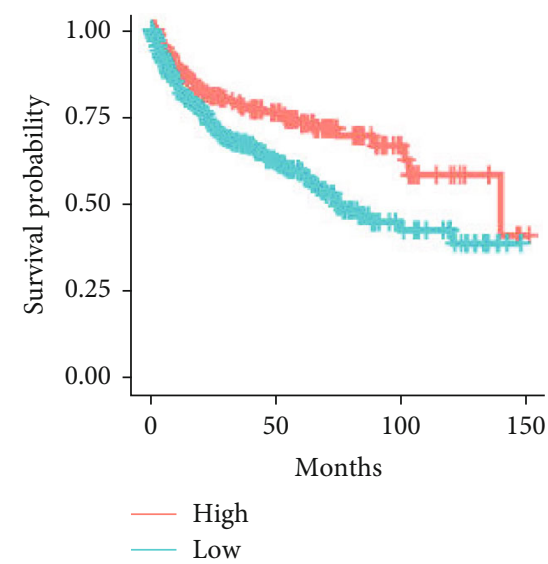

(d)

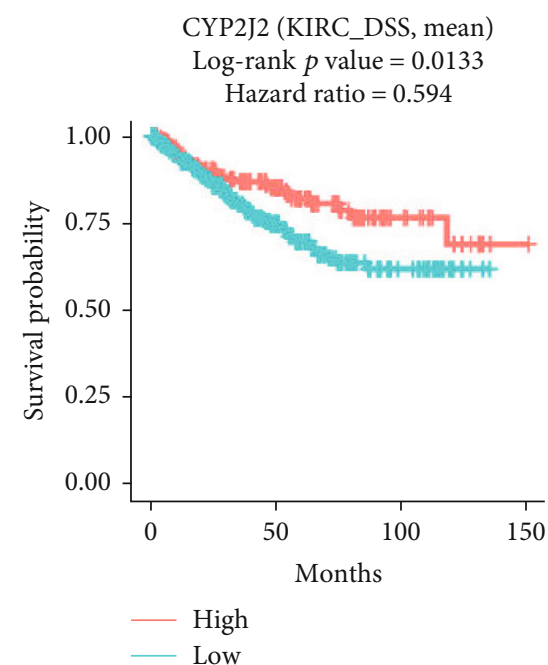

(f)

FIgURE 4: Prognostic value of CYP2J2 expression in KIRC patients was evaluated based on DriverDBv3 database. (a and b) Overexpression of CYP2J2 prolonged OS of KIRC patients. (c-f) Overexpression of CYP2J2 prolonged PFI and DSS of KIRC patients. OS: overall survival; PFI: platinum-free treatment interval; DSS: disease specific survival. 


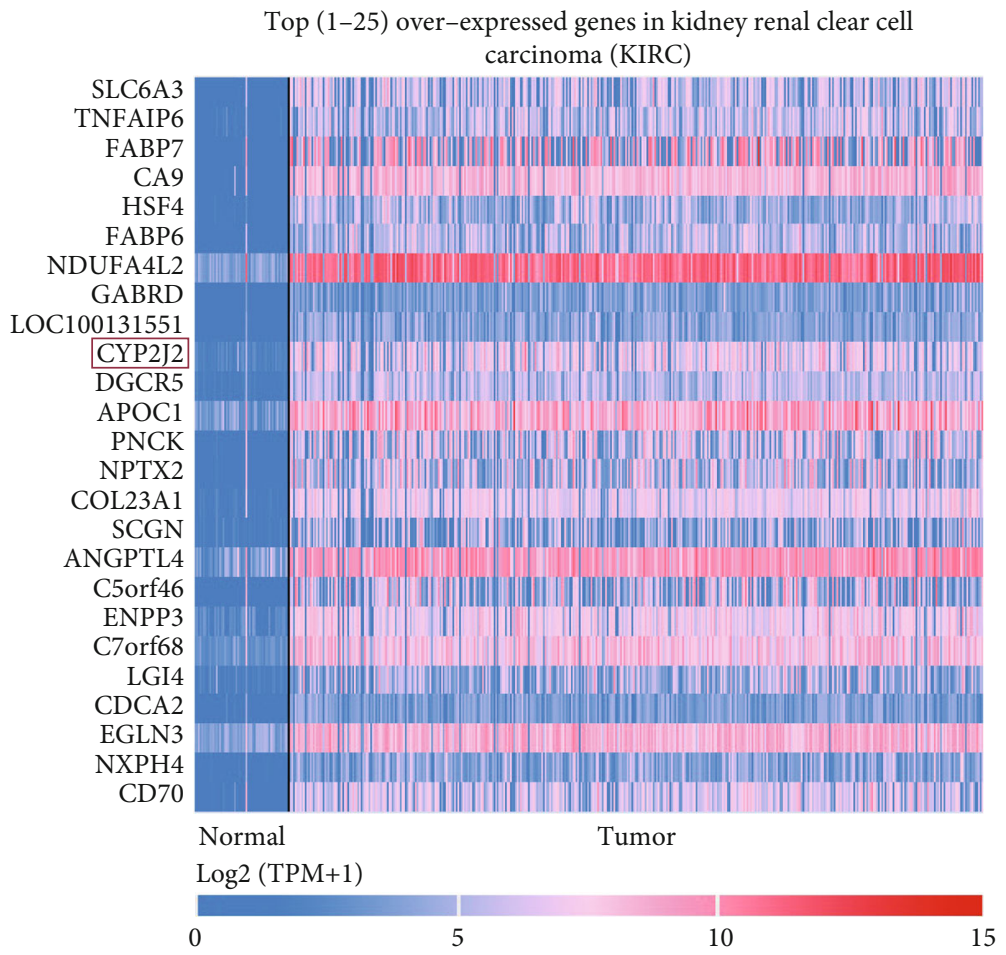

FIGURE 5: CYP2J2 gene is one of the top [1-25] overexpressed genes in KIRC tumor tissues compared to corresponding normal tissues.

2.5. DriverDBv3. DriverDBv3 (http://driverdb.tms.cmu.edu .tw/) is a powerful cancer multiomics database for copy number variations, miRNA expression levels, RNA expression, methylation, and somatic mutations among other clinical data [19]. One of the many functions of the DriverDBv3 database is that it allows users to analyze the association between cancer and genes through "Cancer," "Gene," and "Customized analysis." In this study, DriverDBv3 was used to assess the expression levels of CYP2J2 in different cancer types and to evaluate its prognostic value in KIRC patients. Regarding the survival curve, $p<0.05$ was considered statistically significant.

2.6. GeneMANIA. GeneMANIA (http://genemania.org/) is an easy-to-use website for establishing protein-protein interactions (PPI), protein-DNA interactions, and genetic interactions [20]. In this study, the functions and networks of the CYP2J2 protein were determined through GeneMANIA.

2.7. Metascape and FunRich. Metascape (http://metascape .org/gp/index.html) is an effective tool for comprehensive genomics analysis in the era of big data. It integrates functional enrichment, gene annotation, and interactive group analysis [21]. FunRich (3.1.3 exe) is a user-friendly bioinformatics tool for performing various analyses on generated datasets [22]. We obtained the interactive genes of CYP2J2 through the GeneMANIA network. Then, these genes were inputted into Metascape and FunRich for functional evaluations.

2.8. TIMER. TIMER (https://cistrome.shinyapps.io/timer/) is a simple and practical cancer web server that allows users to evaluate the immunological, genomic, and clinical features of tumors by inputting function-specific parameters [23]. In this study, TIMER was used to determine the expression levels of CYP2J2 in various cancers and to evaluate the correlation between the expression levels of CYP2J2 and immune infiltration levels in KIRC.

\section{Results}

3.1. Expression Levels of CYP2J2 in Different Cancer Types. Using the Oncomine database, which contained total unique analyses of 443 about CYP2J2, we detected diversities in CYP2J2 gene expression profiles between tumor and matched normal tissues. Compared to normal tissues, elevated expression levels of CYP2J2 were observed in bladder, kidney, lung, lymphoma, and ovarian cancer types, while suppressed expression levels of CYP2J2 were observed in cervical, colorectal, esophageal, head and neck, and liver cancer types (Figure 1(a)). Although the expression of CYP2J2 was elevated in a variety of cancers, it was found that elevated CYP2J2 expression levels were most significant in kidney cancer. Then, we analyzed the RNA sequencing data of CYP2J2 through GEPIA. Compared to matched normal tissues, expression levels of CYP2J2 transcripts per million in KIRC were most significant among various tumors (Figure 1(b)). Moreover, expression levels of the CYP2J2 gene in the DriverDBv3 and TIMER database were also the highest in KIRC (Figures 1(c) and 1(d)). These findings showed that the CYP2J2 expression level in KIRC was significantly upregulated compared with normal tissues. Therefore, CYP2J2 may have a potential diagnostic value for KIRC and the correlation between CYP2J2 and KIRC was worth further exploring. 


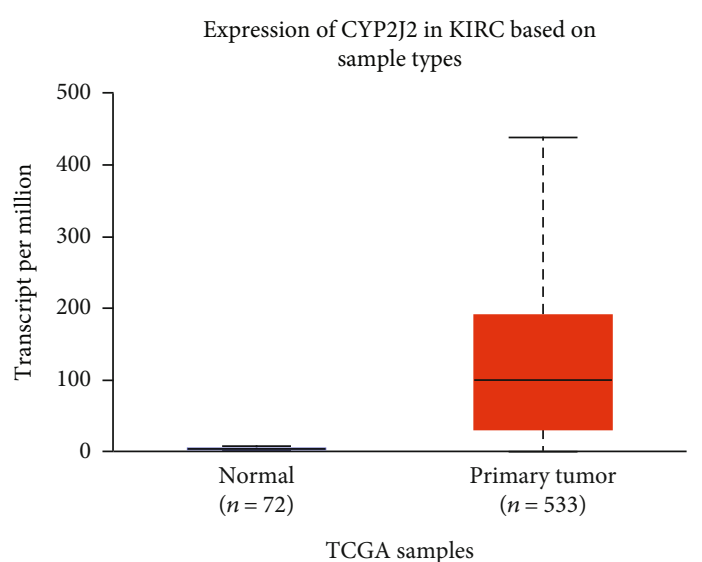

(a)

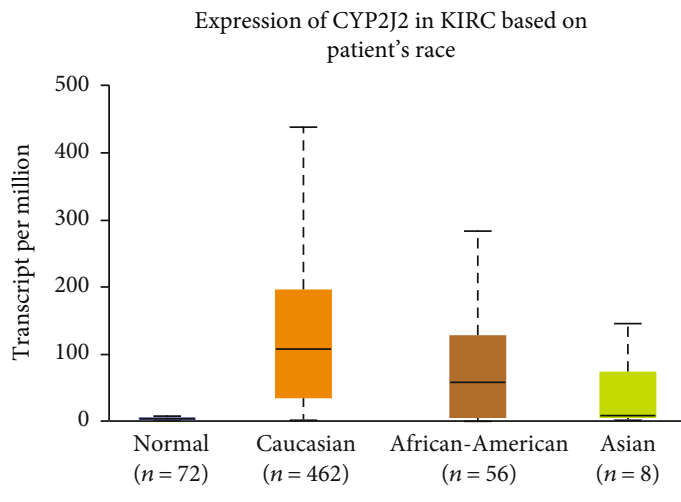

TCGA samples

(c)

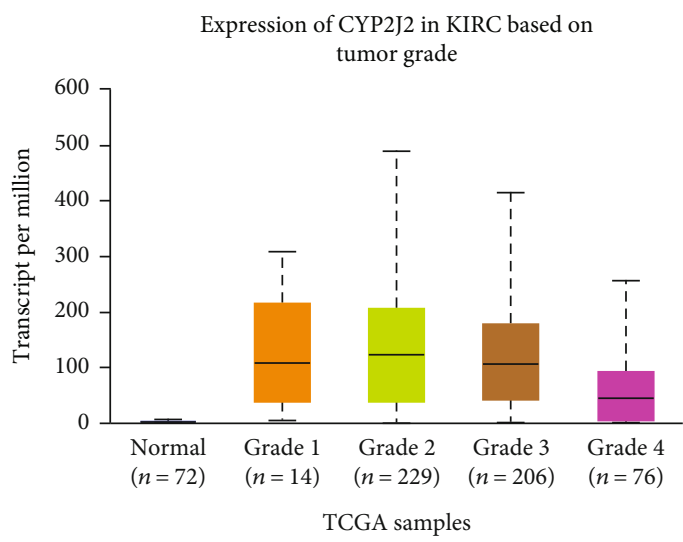

(e)

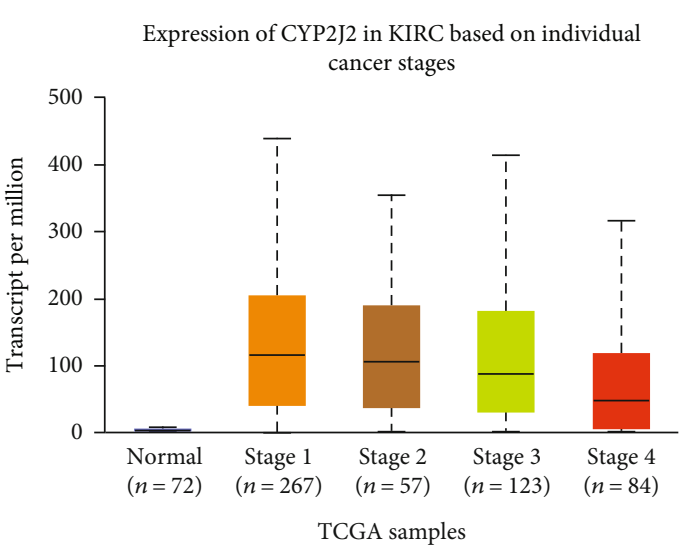

(b)

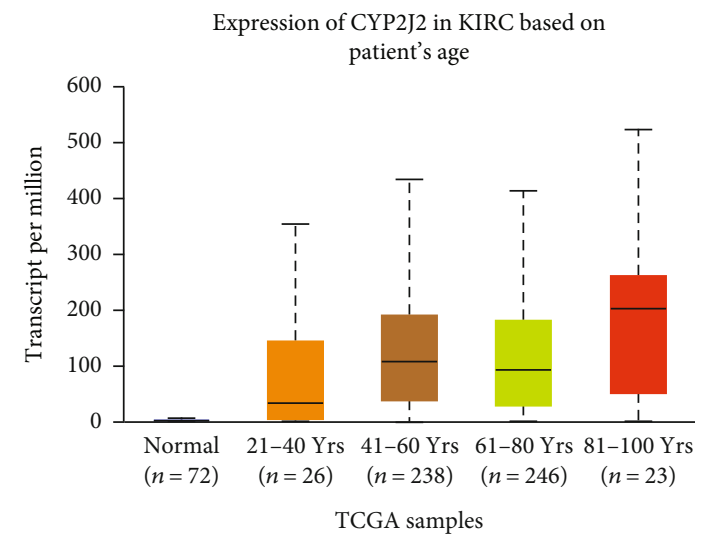

(d)

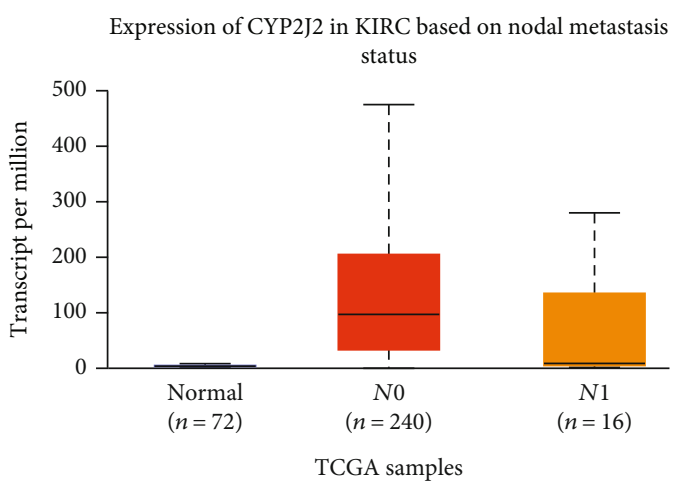

(f)

Figure 6: Expression of CYP2J2 mRNA in KIRC was evaluated by box plot using the UALCAN database. (a-f) Box plots showing the association of sample types, cancer stage, patient's race, age, tumor grade, and nodal metastasis status with CYP2J2 expression in KIRC.

3.2. Potential Prognostic Values of CYP2J2 in KIRC. To determine whether CYP2J2 expression levels are correlated to the prognosis of cancer patients, we evaluated the prognostic value of CYP2J2 in cancer using GEPIA. We found that, even though CYP2J2 expression levels were upregulated or downregulated in various tumor types, CYP2J2 expression levels only had significant correlations with the overall survival time (OS) and disease-free survival (DFS) of KIRC. Moreover, the results indicated that overexpression of CYP2J2 in
KIRC prolonged the OS and DFS (Figure 2). Based on the unique prognostic value of CYP2J2 in KIRC, we further analyzed the relationship between CYP2J2 and KIRC using other databases.

From the UALCAN database, it was found that CYP2J2 overexpression was associated with longer survival outcomes in KIRC patients (Figure 3(a)). Furthermore, we combined the GSE22541, GSE29609, GSE3, and TCGA datasets from OSkirc to verify the effect of CYP2J2 on the survival outcome 


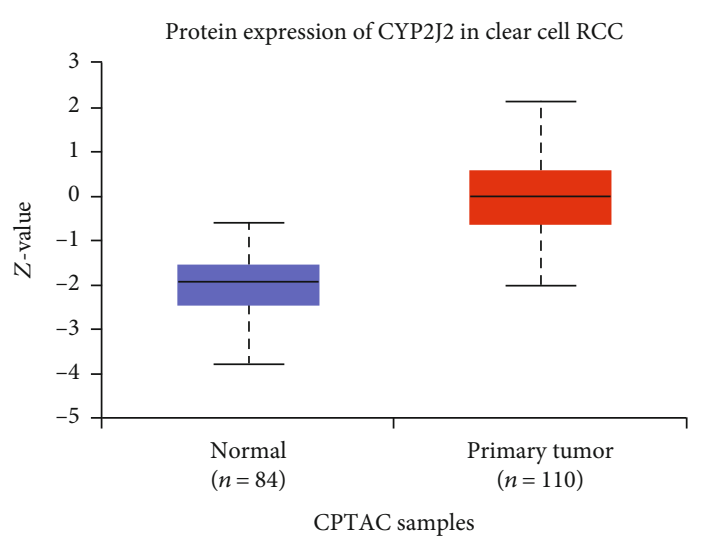

(a)

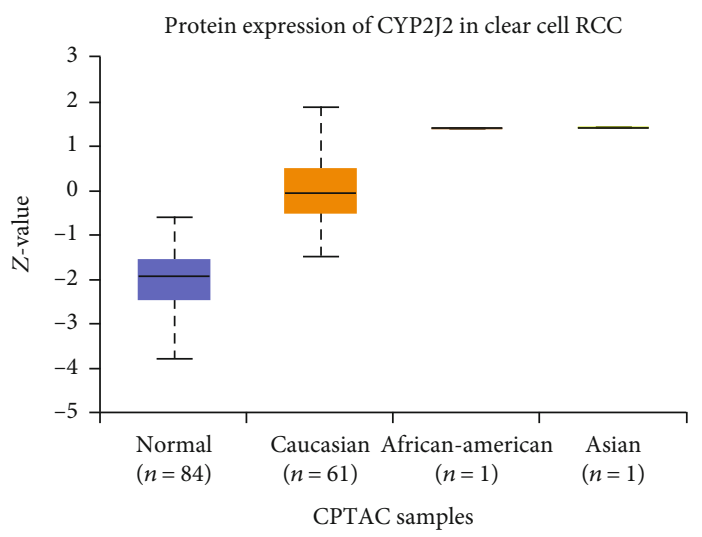

(c)

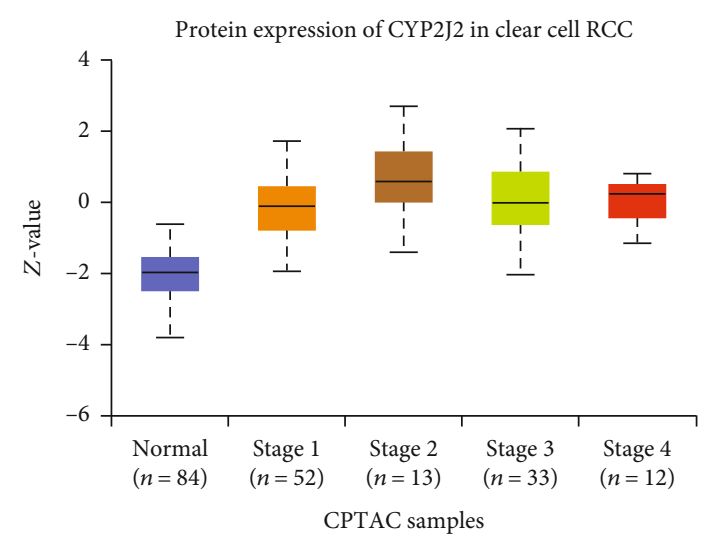

(b)

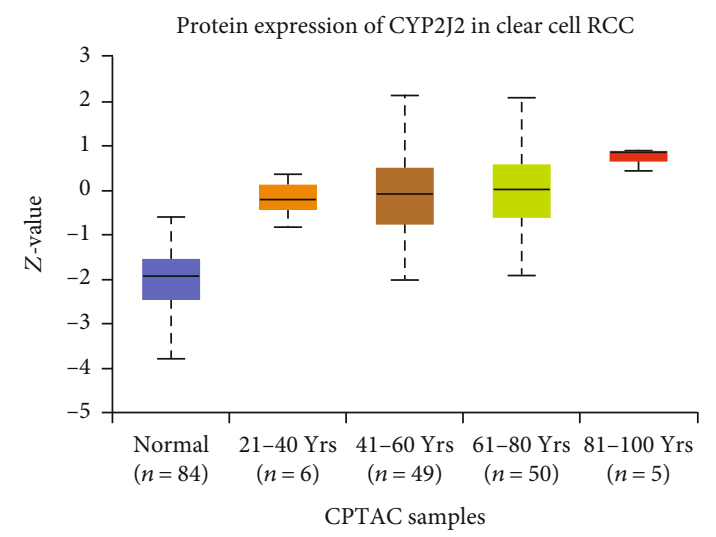

(d)

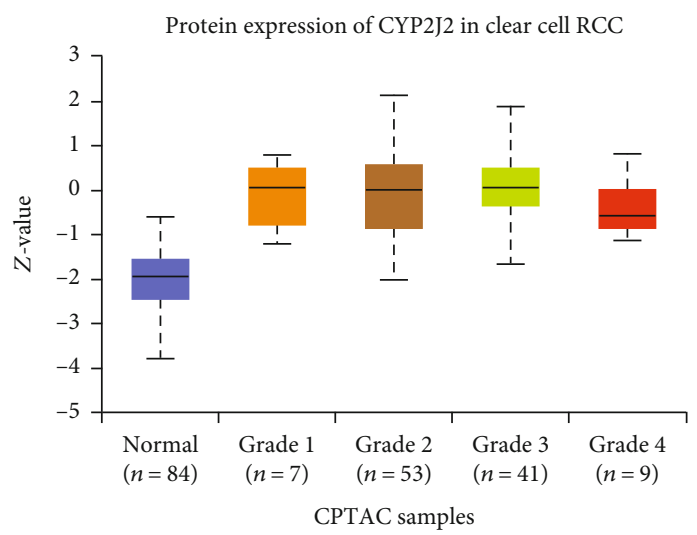

(e)

FIGURE 7: The protein expression level of CYP2J2 in ccRCC was evaluated using box plots in UALCAN database. (a-e) Box plots showing the association of sample types, cancer stage, patient's race, age, and tumor grade with CYP2J2 protein expression level in ccRCC. ccRCC: clear cell renal cell carcinoma; ccRCC=KIRC.

of KIRC patients, and the results also showed that overexpression of CYP2J2 prolonged OS in patients with KIRC (Figures 3(b) and 3(c)). For the DriverDBv3 database, overexpression of CYP2J2 in KIRC predicted better prognostic outcomes when compared to its low expression level (Figure 4), which was basically consistent with the results obtained from GEPIA, UALCAN, and OSkirc databases. Therefore, it can be concluded that the expression levels of CYP2J2 had a very momentous potential prognostic value for KIRC.
3.3. Reanalysis of CYP2J2 Expression in KIRC. To better elucidate on the relationship between CYP2J2 and KIRC, we determined the expression levels of the CYP2J2 gene in KIRC using the UALCAN database. The CYP2J2 gene was one of the top [1-25] overexpressed gene in KIRC (Figure 5), which further indicated that expression levels of the CYP2J2 gene in KIRC tumor tissues were significantly higher than those of normal tissues. Further analyses revealed that the expression levels of the CYP2J2 transcript in KIRC tumor tissues were 


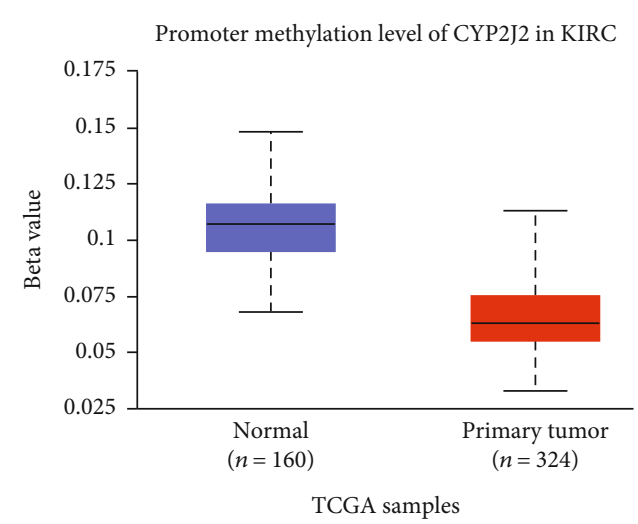

(a)

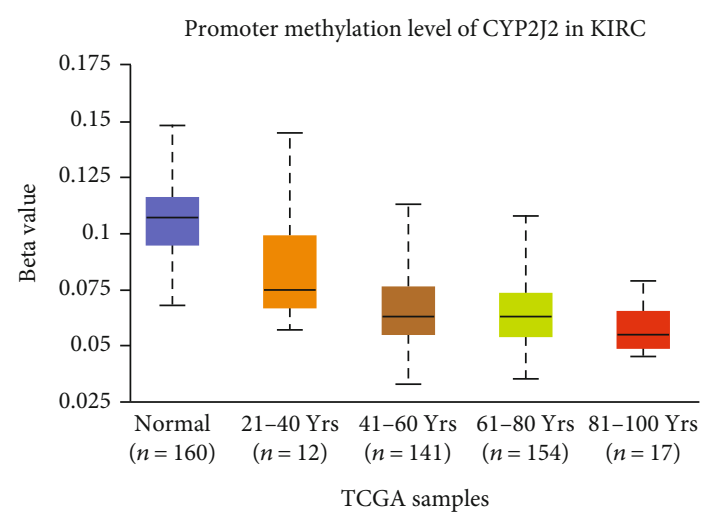

(c)

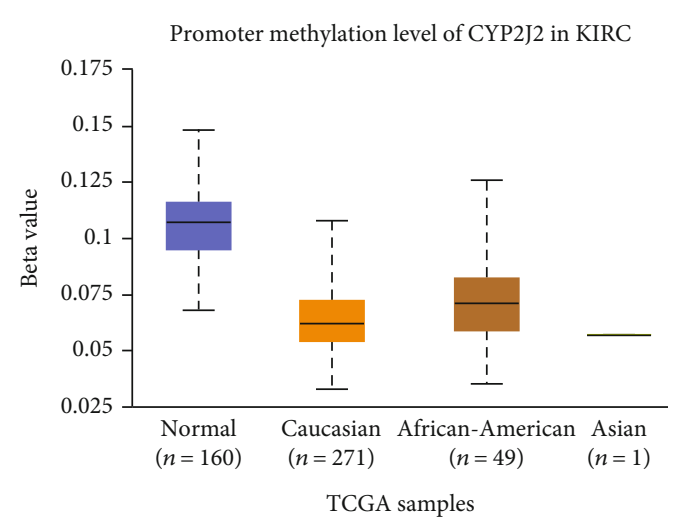

(b)

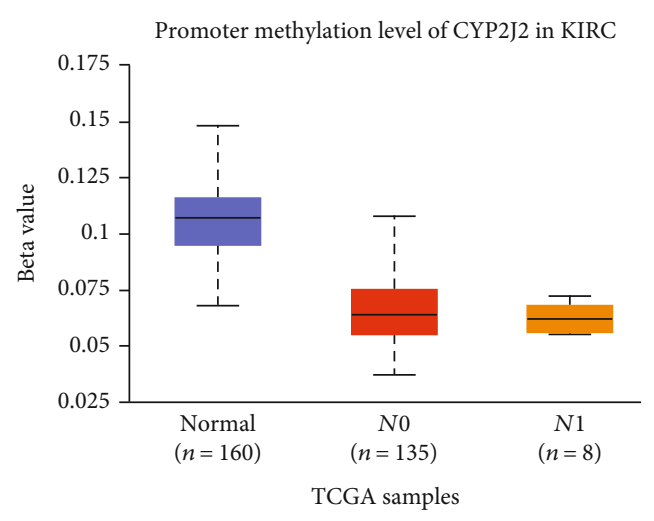

(d)

Figure 8: Promoter methylation levels of CYP2J2 in KIRC were evaluated by box plot using the UALCAN database. (a-d) Box plots showing the association of sample types, patient's race, age, and nodal metastasis status with promoter methylation level of CYP2J2 in KIRC.

significantly higher compared to matched normal samples (Figure 6(a)). Then, the UALCAN database was used to assess various clinical characteristics of KIRC, including cancer stage, patients' race, age, tumor grade, and nodal metastasis status. Further analyses showed that expression levels of the CYP2J2 transcript in KIRC patients were higher in characteristics such as cancer stage 1, Caucasian race, age from 81 to 100 years old, grade 2 , and $N 0$ than other clinical characteristics (Figures 6(b)-6(f)). These findings imply that overexpression of CYP2J2 can be used as an early diagnostic marker of KIRC.

3.4. Potential Diagnostic Values of CYP2J2 Protein Expression in KIRC. Expression levels of the CYP2J2 protein in KIRC were evaluated through the UALCAN database. Compared to matched normal tissues, expression levels of the CYP2J2 protein in KIRC were significantly elevated (Figure $7(\mathrm{a})$ ). From the Oncomine, GEPIA, DriverDBV3, and UALCAN databases, expression levels of the CYP2J2 gene, transcript, and protein in KIRC were significantly elevated compared to matched normal tissues. Furthermore, to investigate the factors mediating the expression of the CYP2J2 protein, we evaluated the correlation between expression levels of the CYP2J2 protein and different clinical characteristics (Figures 7(b)-7(e)), findings of which were basically consistent with the expression trend of the CYP2J2 transcript.
Given the transcript and protein levels of CYP2J2 were significantly elevated in the early stages of KIRC, it was concluded that CYP2J2 can be used as an early diagnostic marker for KIRC.

3.5. Promoter Methylation Level of CYP2J2 in KIRC. We used the UALCAN database to evaluate promoter methylation level of CYP2J2 in KIRC. Compared to normal tissues, the methylation level of the CYP2J2 promoter in KIRC was lower (Figure 8(a)). Moreover, we evaluated the methylation level of the CYP2J2 promoter on the basis of different clinical characteristics, which showed a reverse trend with the expression level of the CYP2J2 transcript and protein (Figures $8(\mathrm{~b})-8(\mathrm{~d})$ ). From the above results, we can infer that elevating the methylation level of the CYP2J2 promoter was likely to downregulate CYP2J2 expression in KIRC.

3.6. PPI Network of CYP2J2. PPI showed functional networks between the CYP2J2 protein and other proteins. CYP2J2 was mainly enriched in responses to xenobiotic stimulus, xenobiotic metabolic process, monooxygenase activity, oxygen binding, steroid metabolic process, and drug metabolic process (Figure 9).

3.7. Functional Enrichment Analyses of CYP2J2. Biological processes of CYP2J2 interactive genes were evaluated by 


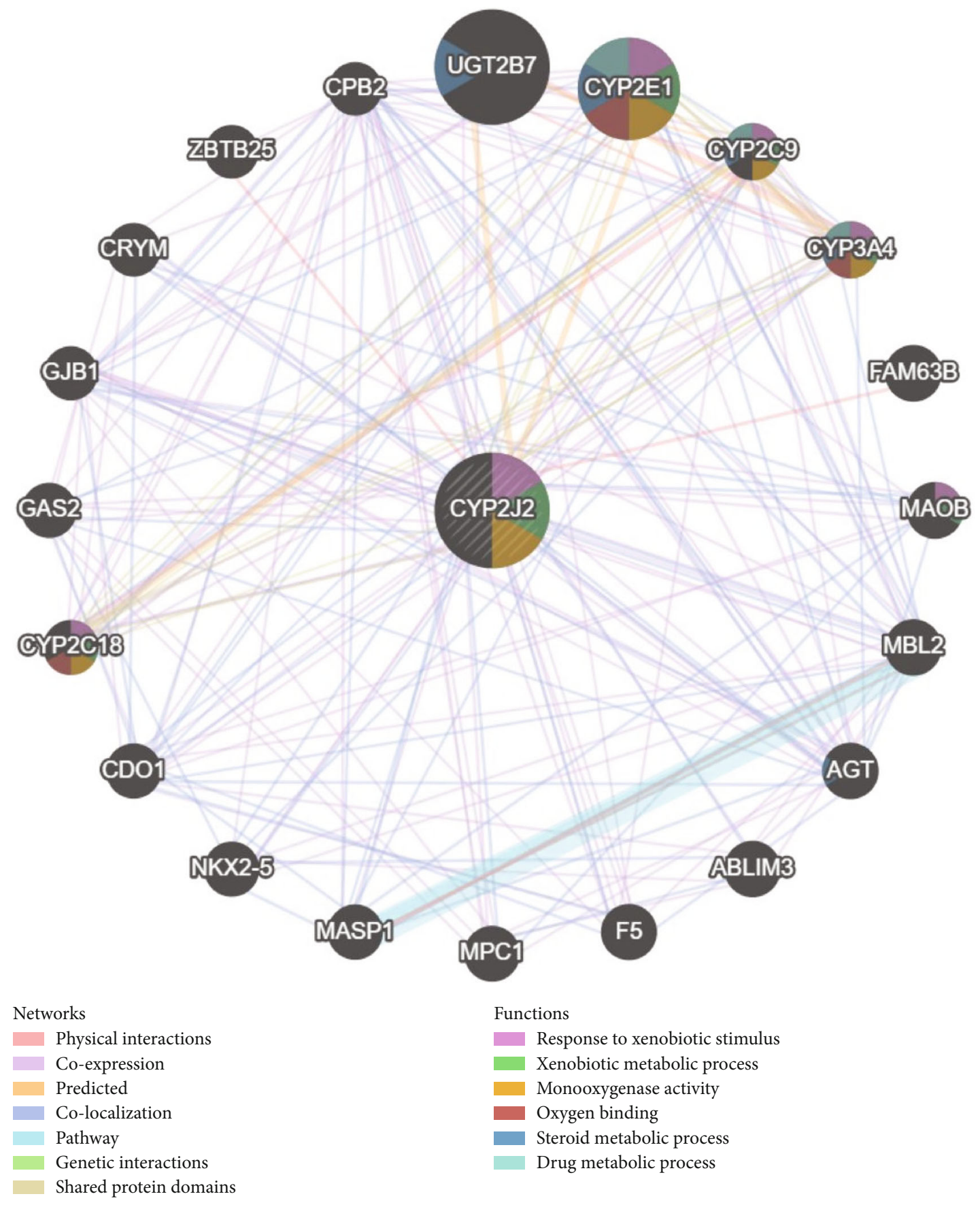

FIGURE 9: The PPI network of CYP2J2 was constructed by GeneMANIA. PPI: protein-protein interaction.

Metascape. We found that response to stimulus, metabolic process, biological regulation, immune system process, multicellular organismal process, cellular component organization or biogenesis, and developmental process was significantly regulated by these genes (Figure 10(a)), implying that CYP2J2 plays a very important role in metabolic process, immune regulation, response to stimulus, and cellular component organization or biogenesis. We also evaluated the biological processes of CYP2J2 interactive genes through FunRich (Figure 10(b)), and findings were very similar to those of Metascape. This further confirmed the biological processes involved in CYP2J2 interactive genes.

3.8. Correlation between CYP2J2 Expression Levels and Immune Cell Infiltration Levels in KIRC. We used the TIMER web server to visualize the correlation between CYP2J2 gene expression levels and immune infiltration levels in KIRC. We found that the expression levels of CYP2J2 were positively correlated with B cells, CD8 + T cells, neutrophil, and dendritic cell infiltration levels in KIRC. However, it was not 


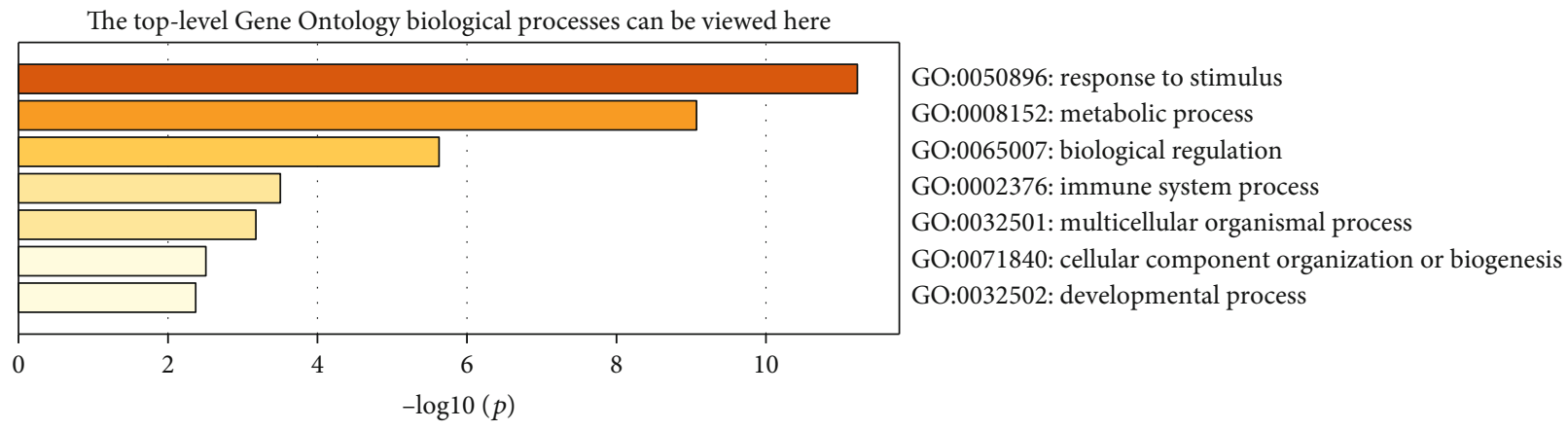

(a)

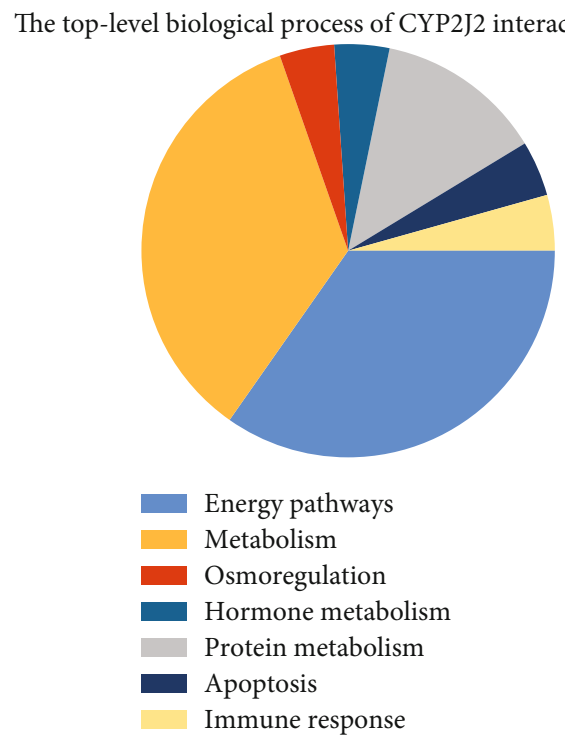

(b)

FIgURE 10: Biological processes involved in CYP2J2 interactive genes. (a) Heat map from Metascape showing the major biological processes involving the CYP2J2 interactive genes. (b) Pie chart from FunRich showing the major biological processes involving CYP2J2 interactive genes.

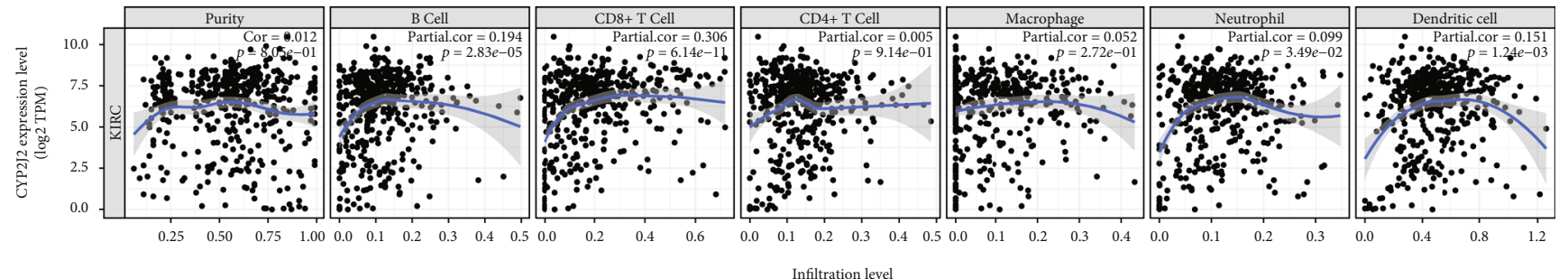

FIGURE 11: Relationships between CYP2J2 expression levels and immune cell infiltration levels in KIRC.

correlated with infiltration levels of CD4 + T cells and macrophages (Figure 11).

\section{Discussion}

Globally, KIRC is one of the most common malignancies, accounting for $80 \%$ of all kidney malignancies [24]. Due to the absence of a clear clinical biomarker for screening KIRC patients, approximately $15 \%$ of KIRC patients have metastatic tumors at the time of diagnosis [25]. Moreover, prognostic outcomes for patients with the same TNM stage and pathological grade may be different [26]. Therefore, it is imperative to identify biomarkers for the early diagnosis of KIRC and to determine their prognosis.

CYP2J2 is a member of the monooxygenase cytochrome P450 family, and it is highly expressed in the endothelium, myocardium, and kidneys [11]. It has been reported that CYP2J2 plays crucial roles in various diseases. For example, overexpression of CYP2J2 is beneficial in the treatment of diabetes [10] and in the reduction of cardiac hypertrophy 
[27]. However, CYP2J2 is significantly expressed in various tumors [28, 29], including KIRC, but it remains unknown if CYP2J2 overexpression in KIRC is beneficial or harmful.

Therefore, we investigated the correlation between CYP2J2 expression and KIRC using various databases, including Oncomine, GEPIA DriverDBv3, and TIMER databases. These databases showed that, among all tumors, CYP2J2 expression levels were significantly elevated in KIRC, implying that CYP2J2 may be a potential diagnostic marker for KIRC. Then, using UALCAN, GEPIA, OSkirc, and DriverDBv3 databases, we evaluated the effect of CYP2J2 expression on the survival time of KIRC patients. We found that overexpression of CYP2J2 prolonged the survival time of KIRC patients. These results suggest that CYP2J2 can be used as an important prognostic marker for KIRC patients. In addition, we reanalyzed the expression levels of the CYP2J2 gene and transcript in KIRC through UALCAN and found that their expression levels were significantly elevated in KIRC tumor tissues than in paracancerous normal samples. Moreover, expression levels of the CYP2J2 transcript in KIRC patients were higher in stage 1 , grade 2 , and $N 0$ clinical features than in other clinical features. The expression trend of the CYP2J2 protein was basically consistent with that of the transcript, which implied that CYP2J2 can be used as an early diagnostic marker for KIRC patients. Some studies have reported that KIRC patients with lower grade, lower stage, and no lymph node metastasis are more likely to have longer survival outcomes than those with other clinical characteristics $[5,30]$, which was in agreement with our results. Taken together, these results indicate that CYP2J2 is a potential biomarker for the early diagnosis and prognosis of KIRC patients. Next, we evaluated the promoter methylation levels of CYP2J2 in KIRC through the UALCAN database, which exhibited a reverse trend with the expression levels of CYP2J2 transcript and protein. The result suggests that an enhanced promoter methylation level of CYP2J2 is likely to downregulate the CYP2J2 expression in KIRC patients.

To further determine the reasons why elevated expression levels of CYP2J2 are beneficial in the prognosis of KIRC patients, we evaluated the PPI network of CYP2J2 through GeneMANIA, while the biological processes involved in CYP2J2 interactive genes were evaluated through Metascape and FunRich. Since CYP2J2 was found to be involved in many biological processes, there were many possibilities for the specific reasons why CYP2J2 overexpression was beneficial in the prognosis of KIRC patients. We found that biological functions of CYP2J2 were correlated with immune processes; therefore, we postulated that this may be one of the reasons as to why KIRC patients with elevated CYP2J2 expression levels have better prognoses. Based on this assumption, we used TIMER to investigate the relationship between expression levels of CYP2J2 and immune cell infiltration levels in KIRC. The expression levels of CYP2J2 were positively correlated with infiltration levels of B cells, CD8 + T cells, neutrophils, and dendritic cells in KIRC. In addition, it has been reported that $\mathrm{B}$ cell infiltration in KIRC can prolong the cancer-specific survival [31], CD8 + T cell infiltration prolonged the OS outcomes of KIRC patients [32], and upregulation of the abundance of neutrophils was associated with a favorable prognosis of KIRC patients [33], while dendritic cells can effectively inhibit tumor recurrence and metastasis [34]. These results suggest that overexpression of CYP2J2 is beneficial to the prognosis of patients with KIRC by regulating the immune microenvironment of the tumor. However, CYP2J2-associated metabolic processes, apoptosis, and stimulus responses may also influence the prognosis of KIRC patients. Even though the mechanism by which CYP2J2 mediates the prognosis of KIRC patients has not been established, our findings suggest that CYP2J2 could be an important marker for the diagnosis and prognosis of KIRC patients and may be a potential therapeutic target for KIRC patients.

\section{Conclusions}

Expression levels of CYP2J2 in KIRC were most significant among various tumors and higher in stage 1 , grade 2 , and N0 clinical features than in other clinical features. Elevated expression of CYP2J2 can prolong survival outcomes in patients with KIRC. The biological processes involved in CYP2J2 interactive genes were significantly correlated with the responses of immune system processes. The expression levels of CYP2J2 were positively correlated with infiltration levels of B cells, CD8 + T cells, neutrophils, and dendritic cells in KIRC. CYP2J2 can be used as an early diagnostic marker and prognostic predictor of KIRC. Mechanistically, overexpression of CYP2J2 improves the prognosis of KIRC patients by regulating the immune microenvironment of the tumor. More studies are needed to confirm our findings and to facilitate the clinical use of CYP2J2 as a prognostic marker or as a therapeutic target for KIRC.

\section{Data Availability}

The datasets analyzed for this study can be found in the Oncomine, GEPIA, DriverDBv3, OSkirc, UALCAN, GeneMANIA, Metascape, FunRich, and TIMER.

\section{Conflicts of Interest}

The authors declare that they have no conflicts of interest.

\section{Authors' Contributions}

(I) Conception and design were done by Xiong Zou. (II) Administrative support was done by Zengnan Mo. (III) Provision of study materials or patients was done by Xiong Zou and Zengnan Mo. (IV) Collection and assembly of data was done by Xiong Zou. (V) Data analysis and interpretation were done by Xiong Zou and Zengnan Mo. (VI) Manuscript writing was done by all authors. (VII) Final approval of manuscript was done by all authors.

\section{Acknowledgments}

This work was supported by grants from the National Key Research and Development Program of China (2017YFC0908000), the Natural Key Research and Development Project (2020YFA0113200), the Major Project of 
Guangxi Innovation Driven (AA18118016), the Guangxi Key Laboratory for Genomic and Personalized Medicine (20-065-33), and Natural Science Foundation of China (81770759, 82060145, and 31970814).

\section{References}

[1] A. Sánchez-Gastaldo, E. Kempf, A. González del Alba, and I. Duran, "Systemic treatment of renal cell cancer: a comprehensive review," Cancer Treatment Reviews, vol. 60, pp. 7789, 2017.

[2] W. Xiao, Z. Xiong, W. Xiong et al., "Melatonin/PGC1A/UCP1 promotes tumor slimming and represses tumor progression by initiating autophagy and lipid browning," Journal of Pineal Research, vol. 67, no. 4, article e12607, 2019.

[3] J. J. Hsieh, M. P. Purdue, S. Signoretti et al., "Renal cell carcinoma," Nature Reviews Disease Primers, vol. 3, no. 1, p. 17009, 2017.

[4] J. Hu, Z. Chen, L. Bao et al., "Single-cell transcriptome analysis reveals intratumoral heterogeneity in ccRCC, which results in different clinical outcomes," Molecular Therapy, vol. 28, no. 7, pp. 1658-1672, 2020.

[5] J. Zheng, L. Wang, Z. Peng, Y. Yang, D. Feng, and J. He, "Low level of PDZ domain containing 1 (PDZK1) predicts poor clinical outcome in patients with clear cell renal cell carcinoma," eBioMedicine, vol. 15, pp. 62-72, 2017.

[6] C. Qin, L. J. Sun, L. Cui et al., "Application of the revised tumour node metastasis (TNM) staging system of clear cell renal cell carcinoma in eastern China: advantages and limitations," Asian Journal of Andrology, vol. 15, no. 4, pp. 550557, 2013.

[7] M. Pichler, G. C. Hutterer, T. F. Chromecki et al., "Predictive ability of the 2002 and 2010 versions of the tumour-nodemetastasis classification system regarding metastasis-free, cancer-specific and overall survival in a European renal cell carcinoma single-centre series," BJU International, vol. 111, no. 4b, pp. E191-E195, 2013.

[8] P. Tamayo, Y. J. Cho, A. Tsherniak et al., "Predicting relapse in patients with medulloblastoma by integrating evidence from clinical and genomic features," Journal of Clinical Oncology, vol. 29, no. 11, pp. 1415-1423, 2011.

[9] J. Ning, T. Liu, P. Dong et al., "Molecular design strategy to construct the near-infrared fluorescent probe for selectively sensing human cytochrome P450 2J2," Journal of the American Chemical Society, vol. 141, no. 2, pp. 1126-1134, 2019.

[10] Y. Yang, R. Dong, Z. Chen et al., "Endothelium-specific CYP2J2 overexpression attenuates age-related insulin resistance," Aging Cell, vol. 17, no. 2, p. e12718, 2018.

[11] X. Xu, X. A. Zhang, and D. W. Wang, "The roles of CYP450 epoxygenases and metabolites, epoxyeicosatrienoic acids, in cardiovascular and malignant diseases," Advanced Drug Delivery Reviews, vol. 63, no. 8, pp. 597-609, 2011.

[12] M. Dai, L. Wu, P. Wang, X. Xu, D. W. Wang, and Z. Wen, "CYP2J2 and its metabolites EETs attenuate insulin resistance via regulating macrophage polarization in adipose tissue," Scientific Reports, vol. 7, no. 1, p. 46743, 2017.

[13] S. W. Park, D. S. Heo, and M. W. Sung, "The shunting of arachidonic acid metabolism to 5-lipoxygenase and cytochrome p450 epoxygenase antagonizes the anti-cancer effect of cyclooxygenase-2 inhibition in head and neck cancer cells," Cellular Oncology (Dordrecht), vol. 35, no. 1, pp. 1-8, 2012.
[14] D. R. Rhodes, S. Kalyana-Sundaram, V. Mahavisno et al., "Oncomine 3.0: genes, pathways, and networks in a collection of 18,000 cancer gene expression profiles," Neoplasia, vol. 9, no. 2, pp. 166-180, 2007.

[15] R. Wei, R. A. Rodrìguez, M. Mullor et al., "Analyzing the prognostic value of DKK1 expression in human cancers based on bioinformatics," Annals of Translational Medicine, vol. 8, no. 8, p. 552, 2020.

[16] Z. Tang, C. Li, B. Kang, G. Gao, C. Li, and Z. Zhang, "GEPIA: a web server for cancer and normal gene expression profiling and interactive analyses," Nucleic Acids Research, vol. 45, no. W1, pp. W98-W102, 2017.

[17] D. S. Chandrashekar, B. Bashel, S. A. H. Balasubramanya et al., "UALCAN: a portal for facilitating tumor subgroup gene expression and survival analyses," Neoplasia, vol. 19, no. 8, pp. 649-658, 2017.

[18] L. Xie, Q. Wang, Y. Dang et al., "OSkirc: a web tool for identifying prognostic biomarkers in kidney renal clear cell carcinoma," Future Oncology, vol. 15, no. 27, pp. 3103-3110, 2019.

[19] S. H. Liu, P. C. Shen, C. Y. Chen et al., "DriverDBv3: a multiomics database for cancer driver gene research," Nucleic Acids Research, vol. 48, pp. D863-DD70, 2019.

[20] D. Warde-Farley, S. L. Donaldson, O. Comes et al., "The GeneMANIA prediction server: biological network integration for gene prioritization and predicting gene function," Nucleic Acids Research, vol. 38, suppl_2, pp. W214-W220, 2010.

[21] Y. Zhou, B. Zhou, L. Pache et al., "Metascape provides a biologist-oriented resource for the analysis of systems-level datasets," Nature Communications, vol. 10, no. 1, p. 1523, 2019.

[22] P. Fonseka, M. Pathan, S. V. Chitti, T. Kang, and S. Mathivanan, "FunRich enables enrichment analysis of OMICs datasets," Journal of Molecular Biology, vol. 433, no. 11, article 166747, 2021.

[23] T. Li, J. Fan, B. Wang et al., "TIMER: a web server for comprehensive analysis of tumor-infiltrating immune cells," Cancer Research, vol. 77, no. 21, pp. e108-e110, 2017.

[24] N. Klümper, D. J. Ralser, E. G. Bawden et al., "LAG3 (LAG-3, CD223) DNA methylation correlates with LAG3 expression by tumor and immune cells, immune cell infiltration, and overall survival in clear cell renal cell carcinoma," Journal for Immunotherapy of Cancer, vol. 8, no. 1, p. e000552, 2020.

[25] R. L. Siegel, K. D. Miller, and A. Jemal, "Cancer statistics, 2018," CA: a Cancer Journal for Clinicians, vol. 68, no. 1, pp. 7-30, 2018.

[26] J. H. Wei, Z. H. Feng, Y. Cao et al., "Predictive value of singlenucleotide polymorphism signature for recurrence in localised renal cell carcinoma: a retrospective analysis and multicentre validation study," The Lancet Oncology, vol. 20, no. 4, pp. 591-600, 2019.

[27] B. Wang, H. Zeng, Z. Wen, C. Chen, and D. W. Wang, "CYP2J2 and its metabolites (epoxyeicosatrienoic acids) attenuate cardiac hypertrophy by activating AMPK $\alpha 2$ and enhancing nuclear translocation of Akt1," Aging Cell, vol. 15, no. 5, pp. 940-952, 2016.

[28] X. Lei, X. Chen, Y. Quan, Y. Tao, and J. Li, “Targeting CYP2J2 to enhance the anti-glioma efficacy of cannabinoid receptor 2 stimulation by inhibiting the pro-angiogenesis function of M2 microglia," Frontiers in Oncology, vol. 10, p. 574277, 2020.

[29] A. Karkhanis, Y. Hong, and E. C. Y. Chan, "Inhibition and inactivation of human CYP2J2: implications in cardiac 
pathophysiology and opportunities in cancer therapy," Biochemical Pharmacology, vol. 135, pp. 12-21, 2017.

[30] Y. Tan, B. Li, F. Peng, G. Gong, and N. Li, "Integrative analysis of sirtuins and their prognostic significance in clear cell renal cell carcinoma," Frontiers in Oncology, vol. 10, p. 218, 2020.

[31] P. J. Stenzel, M. Schindeldecker, K. E. Tagscherer et al., "Prognostic and predictive value of tumor-infiltrating leukocytes and of immune checkpoint molecules PD1 and PDL1 in clear cell renal cell carcinoma," Translational Oncology, vol. 13, no. 2, pp. 336-345, 2020.

[32] S. Zhang, E. Zhang, J. Long et al., "Immune infiltration in renal cell carcinoma," Cancer Science, vol. 110, no. 5, pp. 1564-1572, 2019.

[33] X. Niu, Z. Zhu, E. Shao, and J. Bao, "ACE2 is a prognostic biomarker and associated with immune infiltration in kidney renal clear cell carcinoma: implication for COVID-19," Journal of Oncology, vol. 2021, Article ID 8847307, 15 pages, 2021.

[34] D. S. Lim, J. H. Kim, D. S. Lee, C. H. Yoon, and Y. S. Bae, “DC immunotherapy is highly effective for the inhibition of tumor metastasis or recurrence, although it is not efficient for the eradication of established solid tumors," Cancer Immunology, Immunotherapy, vol. 56, no. 11, pp. 1817-1829, 2007. 